\title{
ENCUESTA
}

\section{Encuesta de aceptación de la revista colombiana de endocrinología, diabetes y metabolismo}

$\mathrm{E}$ l comité editorial de la Revista Colombiana de Endocrinología, Diabetes y Metabolismo, realizó una encuesta a los participantes de los eventos académicos del año 2016 de la Asociación Colombiana de Endocrinología, Diabetes y Metabolismo, ACE, ya que en estos eventos se realizó la entrega de la versión impresa de los cuatro números del Volumen 3 de esta publicación. Esta encuesta se desarrolló con el ánimo conocer el número de personas que reciben nuestra revista, el tipo de profesional que la lee, el grado de aceptación y utilidad en su desempeño profesional, si los lectores la recomiendan a otros profesionales de la salud, además de sugerencias y observaciones.

Se analizaron 470 encuestas, quedando gratamente sorprendidos por una respuesta tan importante ya que en general los colombianos poco tenemos el hábito de contestar este tipo de evaluaciones. Además, como pueden observar en los resultados los lectores que contestaron corresponden en su orden a médicos especialistas (41\%), médicos generales (35\%) es- tudiantes de medicina (12\%) y otros profesionales de la salud $(12 \%)$, lo que está muy de acuerdo a los lineamientos y filosofía de la ACE en cuanto a educación médica continua.

Además estuvimos muy complacidos con su utilidad, ya que esta les ha ayudado a mejorar su desempeño profesional y un gran número la recomendaría a otros colegas. Asimismo, agradecemos las sugerencias planteadas, las cuales se publican para su conocimiento, pues nos ayudan a mejorar nuestra publicación.

Estos resultados nos dan ánimo para seguir trabajando por nuestra asociación y por nuestra revista y sentir que nuestro esfuerzo ha sido útil. Esperamos seguir contando con todos nuestros lectores y lograr posicionar nuestra revista como una de las mejores publicaciones científicas de América Latina.

Enrique Ardila

Editor

\section{Resultados de la encuesta}

\author{
Por favor indique su profesión \\ Respondido: 470 \\ Omitido: 0
}

\begin{tabular}{l|c}
\multicolumn{1}{c|}{ Opciones de respuesta } & Respuestas \\
\hline Estudiante de Medicina & $\mathbf{1 1 , 7 0 \%}$ \\
& 55 \\
\hline Médico General & $35,11 \%$ \\
& 165 \\
\hline Médico Internista & $\mathbf{1 2 , 1 3 \%}$ \\
\hline Médico Endocrinólogo & 57 \\
\hline \multirow{2}{*}{ Médico Ginecólogo } & $2,34 \%$ \\
& 11 \\
Médico Pediatra & $\mathbf{1 , 0 6 \%}$ \\
\hline Profesional otra área de la salud & $\mathbf{1 , 2 8 \%}$ \\
\hline
\end{tabular}


Otra especialidad (por favor especifique)

Epidemiólogo

Auditor y gerente de servicios de salud

Auditoría en salud

Auditoría médica

Bacterióloga

Bacterióloga y laboratorista clínica y

Bacteriología

Bacteriólogo y laboratorista clínico

Biología molecular

Cardiología

Cirugía bariátrica

Control interno y auditoría

Deportóloga

Diabetes

Diabetólogo uba, epidemiologo corpas

Doctora en botecnología

Endocrinólogo pediatra

Enfermera

Enfermera jefe

Enfermera, magisterio en gerontología:

envejecimiento y vejez

Enfermería

Enfermero

Epidemiología

Epidemiólogo

Especialista en gerencia en salud

ocupacional

Estudiante de nutrición y dietética

Estudiante enfermería

Estudiante nutrición y dietética

Gastroenetrología y endoscopía

digestiva

Genetista

Gerencia médica

Homeopatía

Internista cardiólogo

Internista-geriatra

Md eso
Médica familiar

Médica veterinaria, estudiante de doctorado en ciencias farmacéuticas

(diabetes)

Medicina aplicada a la actividad física y

al deporte

Medicina del deporte

Medicina del trabajo

Medicina educación física y deporte

Medicina familiar

Medicina laboral

Medicina trabajo docente en geriatría y gerontologia

Médico cardiólogo

Médico deportólogo

Medico ESP en salud ocupacional

Médico familiar

Médico farmacólogo

Médico laboral

Médico y cirujano con maestría en ciencias básicas médicas-farmacología

Medicina familiar

Neurología

Nutrición

Nutrición y dietética

Nutricionista

Nutricionista dietista

Nutrióloga

Ortopedista

Patología

Pediatra diabetóloga

Psicología

Psiquiatra

Residente medicina familiar

Salubrista, MS SSR

Salud familiar

Salud ocupacional

Trabajadora social
$24,47 \%$

115

¿Usted ha leído la Revista Colombiana de Endocrinología, Diabetes y Metabolismo?

Respondido: 470

Omitido: 0

$\mathrm{Si}$

$69,36 \%$

326

No

$30,64 \%$

144

Total

470 


\section{Si la respuesta anterior fue positiva, como accedió a la Revista Colombiana de Endocrinología, Diabetes y Metabolismo \\ Respondido: 331 \\ Omitido: 139}

\begin{tabular}{|c|c|c|}
\hline \multicolumn{2}{|c|}{ Opciones de respuesta } & Respuestas \\
\hline \multicolumn{2}{|c|}{ En un evento de la Asociación Colombiana de Endocrinología } & $\begin{array}{c}\mathbf{6 7}, 37 \% \\
223 \\
\end{array}$ \\
\hline \multicolumn{2}{|l|}{ En internet } & $\begin{array}{c}27,49 \% \\
91\end{array}$ \\
\hline \multicolumn{2}{|c|}{ Otro medio (por favor especifique) } & $\begin{array}{c}5,14 \% \\
17\end{array}$ \\
\hline $\begin{array}{l}\text { A través de colega } \\
\text { En físico } \\
\text { La conocí en Simposio } \\
\text { Me la prestaron } \\
\text { Me llega a la casa en físico }\end{array}$ & $\begin{array}{l}\text { Me llega a la oficina } \\
\text { Por obsequios en eventos } \\
\text { Préstamo } \\
\text { Universidad } \\
\text { Visita médica }\end{array}$ & \\
\hline Total & & 331 \\
\hline
\end{tabular}

\section{¿Recuerda algún artículo de la Revista que le haya gustado? \\ Respondido: 362 \\ Omitido: 108}

\begin{tabular}{|c|c|}
\hline Opciones de respuesta & Respuestas \\
\hline $\mathrm{Si}$ & $\begin{array}{c}\mathbf{4 2 , 8 2 \%} \\
155\end{array}$ \\
\hline No & $\begin{array}{c}\mathbf{5 6 , 9 1 \%} \\
206 \\
\end{array}$ \\
\hline Si su respuesta fue positiva ¿A cuál artículo se refiere? & $\begin{array}{c}\mathbf{3 6 , 1 9} \% \\
131\end{array}$ \\
\hline $\begin{array}{l}\text { Menopausia y metabolismo óseo } \\
\text { ¿Es mandatoria la tamización de disfunción tiroidea en toda mujer en la consulta } \\
\text { preconcepcional?: Aportes de un estudio local } \\
\text { Progresos en la terapia del cáncer avanzado de tiroides, bases fisiopatológicas. } \\
\text { Epidemiología de la retinopatía diabética y su relación con la diabetes. } \\
\text { ADA 2015, actualización } \\
\text { Aporte colombiano a la tiroidología } \\
\text { Cambios terapéuticos en el estilo de vida para patologías endocrinológicas } \\
\text { Casos clínicos de acromegalia } \\
\text { Casos diabetes y artículos endocrino muy buenos evidencia } \\
\text { Conteo de carbohidratos para diabéticos } \\
\text { Controversias actuales en el diagnóstico de la diabetes mellitus gestacional } \\
\text { Controversias en diabetes gestacional y uso de antidiabéticos orales en la gestación } \\
\text { Correlación entre valores de la glucemia basal y la PTOG en niños con sobrepeso } \\
\text { Creo que el artículo tenia que ver con medicamentos endocrinos por mi profesión } \\
\text { Cushing } \\
\text { Depresión y ansiedad } \\
\text { Después de los bifosfonatos que terapia utilizamos? Teriparatide, Denosumab }\end{array}$ & \\
\hline
\end{tabular}


Diabetes

Diabetes en la era preinsulínica

Diabetes insulinoterapia

Diabetes mellitus

Diabetes y la depresión

Diabetes y obesidad

Diagnóstico diabetes gestacional

Diagnóstico y manejo de la hiponatremia en adultos. Énfasis en síndrome de secreción inadecuada de hormona antidiurética

Dislipidemia y mas

Dosis de levotiroxina varía según la etiología del hipotiroidismo y el peso corporal Cáncer de Tiroides

El manejo de heridas varicosas

El papel de la vitamina D en la gestación y en la preeclampsia: de la biología molecular a la clínica

Enfermedades tiroideas

Es necesaria la medición de la T3 para el diagnóstico de hipotiroidismo primario? Evaluación de causas secundarias de baja masa ósea en mujeres colombianas con osteoporosis posmenopáusica

Evaluación global de la obesidad, menopausia

Exposición a la luz nocturna, disrupción del ritmo cardiano y la obesidad

Feocromocitoma

GPC para el DX, tratamiento y seguimiento de la DM tipo 1 en la población mayor de 25 años

Guía para Dx y seguimientos de DM1 en adultos

Guía práctica clínica para el diagnóstico y tratamiento DM tipo 1

Guías manejo DM

Epidemiología de toroides

Hipercalcemia maligna

Hiperparatiroidismo

Hipertension arterial pulmonar

Hipertiroidismo

Hiponadrenia

Hipopituitarismo posterior a lesión traumática

Hipotiroidismo

Historia de la Endocrinología

HTA

Insulinoterapia y actualidad

Interpretación de pruebas de función tiroidea

La atención especializada en diabetes reduce los costos directos del pte DM

Lo nuevo sobre diabetes

Tiroides

Manejo de DM

Manejo de estatinas

Manejo del feocromocitoma 10 años de experiencia

Mutación BRAF en pacientes con ca de tiroides

Regulación del eje y la menstruación

Nuevos avances en diabetes tipo II

Nutrición en pacientes con Diabetes mellitus

Obesidad

Obesidad y diabetes 
Obesidad, dislipidemia, cirugía bariátrica

Obesidad en niños

Papel de la vitamina D en la gestación y la preeclamsia

Patología tiroidea

Pie risk, una herramienta para la prevención del pie diabético

Predicción del tamaño de prolactinomas mediante el valor plasmático de prolactina

Primer transplante de islotes realizado en Colombia, experiencia fundación Valle de Lili

Progresos en la terapia del cáncer avanzado de tiroides

Proyecto de vida nueva en Barranquilla detección temprana de la diabetes

Referente a Levotiroxina y Hipotiroidismo

Reporte de caso de Síndrome de Gordon

Resumen de congreso

Revisión de hiponatremia, actualización 2016

Revisión Vitamina D

Síndrome de Mauriac

Síndrome metabólico.- Manejo de la diabetes mellitus

Tamización obligatoria de disfunción tiroidea preconcepcional

Tiroides

Tiroides y embarazo

Tiroiditis

Tormenta tiroidea

Trastornos autoinmunes tiroides

Urgencias endocrinológicas

Valores de pruebas tiroideas (TSH y T4 libre) en una población adulta de la ciudad de Medellín, Colombia

Vitamina D en Colombia, osteoporosis.

Vitamina D y salud ósea en la mujer posmenopáusica

\section{¿Ha leído un número completo de la Revista?}

\section{Respondido: 404}

Omitido: 66

\begin{tabular}{l|c}
\multicolumn{1}{c|}{ Opciones de respuesta } & Respuestas \\
Si & $38,86 \%$ \\
& 157 \\
\hline \multirow{2}{*}{ No } & $61,14 \%$ \\
& 247 \\
\hline Total & 404 \\
\hline
\end{tabular}

\section{¿Esta revista le ha ayudado en su formación profesional y en el manejo de sus pacientes?}

\section{Respondido: 371}

Omitido: 99

\begin{tabular}{l|c}
\multicolumn{1}{c|}{ Opciones de respuesta } & Respuestas \\
Si & $\mathbf{8 5 , 9 8 \%}$ \\
& 319 \\
\hline No & $\mathbf{1 4 , 0 2 \%}$ \\
\hline Total & 52 \\
\hline
\end{tabular}




\section{¿La recomendaría a otros colegas?}

Respondido: 367

Omitido: 103

\begin{tabular}{l|c} 
& Respuestas \\
Si & $93,19 \%$ \\
& 342 \\
\hline \multirow{2}{*}{ No } & $6,81 \%$ \\
Total & 25 \\
\hline
\end{tabular}

\section{Comentarios adicionales}

Excelente programación de eventos que contribuyen mejorar educación continuada de los profesionales de la salud.

Actualizaciones periódicas que nos permiten tener aportes actualizados a nuestra profesión y así mantener a la vanguardia.

Agradecería contar con la revista por tener un contenido muy enriquecedor.

Agradecerles por su trabajos e investigaciones además de la gran ayuda que nos brindan tanto a través de la revista como lo eventos de actualización. FELICITACIONES.

Agradezco por tan beneficioso aporte a mis conocimientos y profesión.

Aunque conozco la revista, he leído solo artículos que me han llamado la atención y los considero de gran interés en el área de la endocrinología, y si la he recomendado a mis colegas.

Buenas tardes, reciban cordial saludo y un agradecimiento a su formación, es muy grato contar con su medio físico y de programas de formación, he leído algunos artículos de su revista, entregada en el simposio en Armenia. Muchísimas gracias por su apoyo de educación continuada, ojalá se continúen con estos eventos y programas.

Comentarios y análisis de guías y artículos relevantes de otros autores u revistas.

Como director médico de la fundación hospital San José de la ciudad de Buga, quiero suscribirme, nosotros somos un centro que ademas tiene estudiantes de medicina y medicos internos de 3 universidades, que son: universidad libre seccional Cali, Universidad Javeriana Cali, Universidad UCEVA de Tulúa.

Como médico general aprecio la labor constante, permanente, en mantener actualizados a los médicos generales.

Creo es la única asociación que verdaderamente hace educación continuada y elimina barreras de acceso a la misma.

Deberían por lo menos los titulares darlos a conocer mes a mes a los diferentes correos para mayor difusión de la revista.

Deberían salir más artículos del día, día del médico general gracias.

Deseo felicitarlos por tan alta calidad de sus publicaciones, espero que pronto sea una revista indexada.

Dinámica para leer temas interesantes para todo público y especialidades

Doy gracias a Dios por esta sociedad la cual reconozco como una de las más organizada del país. Yo labora en una unidad neonatal pero me apasiona ir así eventos y cada día crezco como profesional ya que como médico general no solo puedo quedarme con conceptos de pediatría y ustedes me retroalimentan. Gracias por darme la posibilidad de ser un mejor médico para mis pacientes.

El trabajo de la asociación de endocrinología, diabetes y metabolismo es realmente enriquecedor. Actualiza mis conocimientos en todo momento.

Es muy buena revista con lo necesario de cada tema, sigan así.

Es muy buena y completa, la información producto de sus investigaciones aportan al mejoramiento del cuidado en los pacientes.

Es muy educativa.

Es un excelente medio para adquirir conocimientos y fortalecer el aprendizaje.

Es una excelente publicación.

Estaré pendiente ya que el estudio Empa reg Outcome trae datos muy interesantes para cardiología.

Excelente contenido científico.

Excelente en su formato y contenido.

Excelente para estar actualizado.

Excelente publicación que ayuda a recordar algunas cosas y otros nuevos aprendizajes.

Excelente revista sobre todo los temas de Insulinoterapia.

Excelentes publicaciones espero obtener nueva información para el manejo de pacientes con enfermedades metabólicas.

Excelentes temas para actualización.

Felicitaciones por la publicación.

Felicitaciones, las actividades y producciones de su asociación son excelentes.

Felicitarlos por tan completa información brindada en dicha revista! Felicito a la asociación por ese esfuerzo que hacen para actualizar la comunidad medica en general.

Gracias por los aportes de conocimiento que nos da la sociedad de endocrinología.

Gracias por su perseverancia!, Felicitaciones!

Gracias por tener en cuanta a los médicos que laboramos en primer nivel de atención. 
He comprado algunos de los libros que se ofrecen en los talleres y eventos, claro queme encantaría saber sobres los temas que se han publicado.

Incentiva al medico a escribir, falta mucha investigación.

La asociación es líder en multiplicación de la información a través de varios canales de comunicación, los felicito por eso, no es muy frecuente en Colombia para nosotros los médicos.

La empleo como documento guía para el desarrollo de actividades prácticas con mis estudiantes de Bioquímica del programa de Medicina de la universidad Surcolombiana, en Neiva.

La endocrinología como la medicina deportiva van de la mano deberían hacer mas artículos referentes y sobre todo en hormonas y deporte.

La revista es muy buena y el rigor exigido para los artículos es alto, lo que redunda en gran calidad

La revista esta bien. Junto con los eventos. Y las video clases virtuales.

Las charlas son muy especificas y temas importantes de diabetes y endocrinología.

Le he ojeado y me parece interesante.

Los felicito por el esfuerzo hecho.

Los tópicos clínicos que aquí se depuran decantando los conceptos clínicos y fisiológicos del sistema endocrino son de interés clínico.

Me dispondré a leer los números disponibles. Lo que si les digo es que el año 2016 ustedes realizaron excelentes congresos y simposios (participe en 4 de ellos)

Me gusta la diagramación y los temas.

Me gusta la revista y los eventos que realizan de congresos y seminarios con muy buenos temas de actualidad, y muy buenos especialistas.

Me gusta muchos sus eventos espero que me tengan en cuenta siempre. Me gustaría conocer mas artículos sobre infarto agudo del miocardio. Me gustaría conocerla

Me gustaría que hubiera la posibilidad de conocerla un poco mas fácil, en realidad desconozco si esta la posibilidad de leer artículos en linea.

Me gustaría que me enviaran la revista, considero que la sociedad colombiana de endocrinología es una asociación muy respetable.

Me gustaría recibir más invitaciones a charlas, congresos, actividades. Gracias por contribuir al aprendizaje.

Me ha servido para docencia en la facultad.

Me interesa continuar leyéndola, así sea en números atrasados.

Me parece muy buena y ayuda a la educación médica continuada.

Me parece que sus temas son interesantes, y bien explicados, no tengo queja alguna, me encanta su revista.

Me parece un gran herramienta de formación medica, por lo que me gustaría subscribirme para poder revisar cada ejemplar nuevo. Es un material de calidad.

Me gusta el tema de diabetes y tiroides somos una clínica de heridas debo estar actualizada para el manejo de mis pacientes.

Mil gracias por mantenernos actualizados, yo soy médico educador en diabetes y manejo pacientes con riesgo cardiovascular.

Mis mas sinceras felicitaciones y agradecimiento por permitirnos disfrutarla.
Muchas gracias por tenerme en cuenta. La revista esta a la vanguardia en temas y conocimientos para los profesionales que nos interesamos en los temas de endocrinología.

Muy actualizada con un alto nivel académico.

Muy buen aporte a los profesionales información confiable y científica con buenos argumentos. Muy interesante.

Muy importante las capacitaciones.

Muy organizados y buenos ponentes

No cambiar su manejo y temática.

No conozco la revista, pero si tengo interés en ella porque laboro en el programa de pacientes cronicod.

No conozco sobre la revista solo me han enviado sobre eventos de congresos en Barranquilla me gustaría conocer la revista y poder leer los artículos.

No he leído la revista por tanto no puedo responder todas las preguntas pero pienso que debe ser de muy buena calidad como todo lo que hace la asociación en materia de docencia.

Perfecto manejo del lenguaje técnico que permite el fácil entendimiento y comprensión de la información, dinámico diseño, presentación fácil de llevar con uno.

Que continúen con esta labor de educación médica continua. Ya que es la única posibilidad de estar al tanto de los adelantos y nuevas perspectivas de tratamiento. Muchas gracias.

Que se motive para que otras especialidades participen en la revista con la publicación de trabajos o artículos relacionados.

Reconozco la labor académica que vienen adelantando. Muy adecuada.

Revista adecuada para conocimiento científico. Con estudio colombiano que nos hace tener una buena aplicación clínica en nuestro entorno.

Revista buena, y completa, variada y con información sobre eventos académicos.

Sería bueno incluir información sobre creación de nuevos hábitos desde PNL o terapias conductuales cognitivas.

Seria importante que se permitiera la publicación de artículos de profesionales en otras áreas incluyendo investigación básica.

Seria prudente un artículo sobre nuevos métodos diagnosticos en endocrinología

Sigan adelante con la publicación.

Sigan con esa labor académico, investigativa y clínica que es un buen apoyo en todos los niveles.

Son artículos buenos con evidencia científica.

Son medios que ayudan a la educación continua.

Sus contenidos teóricos son muy prácticos y exactos.

Tiene publicaciones interesantes debería tener mayor difusión.

Todo excelente. 